\title{
Correlation between cosmic ray intensity variations observed by the large acceptance GRAPES-3 muon telescope and interplanetary magnetic field.
}

H. Kojima ${ }^{a, b}$, S. Shibata ${ }^{b}$, A. Oshima ${ }^{* b}$, S. Kawakami ${ }^{c}$, Y. Hayashi' ${ }^{c}$ I. Morishita ${ }^{d}$, T. Nakamura $^{e}$, T. Nonaka ${ }^{f}$, S. Ogio ${ }^{c}$, H. Takamaru ${ }^{b}$, K. Tanaka ${ }^{g}$, N. Ito ${ }^{c}$, T. Matsuyama ${ }^{c}$, K. Yamazaki ${ }^{h}$, M. Tokumaru' ${ }^{i}$, S. K. Gupta ${ }^{j}$, S. R. Dugad $^{j}$, B. Hariharan ${ }^{j}$, P. Jagadeesan $^{j}$, A. Jain ${ }^{j}$, V. B. Jhansi ${ }^{j}$, P. K. Mohanty ${ }^{j}$, S. D. Morris $^{j}$, P. K. Nayak ${ }^{j}$, P. S. Rakshe ${ }^{j}$, K. Ramesh ${ }^{j}$, B. S. Rao ${ }^{j}$, L. V. Reddy ${ }^{j}$, M. Zuberi ${ }^{j}$, S. Ahmad $^{k}$, A. Chandra $^{k}$ and P. Subramanian ${ }^{l}$

${ }^{a}$ Faculty of Engineering, Aichi Institute of Technology, Toyota, Aichi, Japan

${ }^{b}$ Faculty of Engineering, Chubu University, Kasugai, Aichi, Japan

${ }^{c}$ Graduate School of Science, Osaka City University, Osaka, Japan

${ }^{d}$ School of Health Sciences, Asahi University, Mizuho, Gifu, Japan

${ }^{e}$ Faculty of Science, Kochi University, Kochi, Japan

${ }^{f}$ Institute for Cosmic Ray Research, University of Tokyo, Kashiwa, Chiba, Japan

${ }^{g}$ Graduate School of Information Sciences, Hiroshima City University, Hiroshima, Japan

${ }^{h}$ Kanagawa University, Kanagawa, Japan

${ }^{i}$ Institute for Space Science and Environment, Nagoya University, Nagoya, Aichi, Japan

${ }^{j}$ Tata Institute of Fundamental Research, Mumbai, India

${ }^{k}$ Aligarh Muslim University, Aligarh, India

${ }^{l}$ Indian Institute of Science Education and Research, Pune, India

E-mail: jh1cvo@gmail.com

\begin{abstract}
The variations of the cosmic ray intensity observed at the Earth reflect the various solar activities through the variations of solar wind plasma that fills up the heliosphere. Thus, the correlation studies between cosmic ray intensity variations and the interplanetary plasma parameters, such as the solar wind speed, the direction, and the strength of interplanetary magnetic field (IMF), are expected to provide important information on the space weather near the Earth.

We have observed the cosmic ray intensity with the large acceptance GRAPES-3 muon telescope, located at Ooty in south India since 2000. We have obtained interesting results concerning the diffusion coefficient of cosmic rays near the Earth from the regression analysis with the solar wind speed [Kojima et al., Phys. Rev. D 98 (2018) 022004].

In this conference, we will report the results of the regression analysis of the cosmic ray intensity variations with the IMF.
\end{abstract}

36th International Cosmic Ray Conference -ICRC2019-

July 24th-August 1st, 2019

Madison, WI, U.S.A.

* Speaker. 


\section{Introduction}

We have obtained the relations (Regression coefficients) between the variations of the cosmic ray intensity observed by the large acceptance GRAPES-3 muon telescope (G3MT, $560 \mathrm{~m}^{2}$ ) [1] at Ooty $\left(11.4^{\circ} \mathrm{N}\right.$ latitude, $76.7^{\circ} \mathrm{E}$ longitude and $2200 \mathrm{~m}$ altitude) in India and the variations of the solar wind velocity [2]. We also have obtained the radial density gradient of Cosmic rays near the Earth from the amplitudes of phase reversal of Cosmic ray sidereal anisotropies on the polarity of the interplanetary magnetic field (IMF) [3][4][5]. From these quantities and the one dimensional Parker's diffusion convection equation [6] which explains the propagation of Cosmic rays in the Heliosphere, we deduced the diffusion coefficient of the Cosmic rays in the IMF plasma [7]. Then, we can explain the role of the variation of the solar wind velocity on the propagation process of the Cosmic rays in the Heliosphere. Our next step of the investigation is to bring out the actual processes which determine the value of the diffusion coefficient $(\kappa)$.

In the process of the Cosmic ray propagation (diffusion and convection), Cosmic ray particles collide not with plasma particles but with irregularities of IMF frozen in the plasma. So, properties of IMF would determine the value of diffusion coefficient. In this paper, we try to analyze the relationships between Cosmic ray intensity variations $\left(\Delta I_{\mu}\right)$ observed by G3MT and three parameters of IMF supplied by OMNI2 data base [8] : solar wind velocity $\left(\Delta V_{\mathrm{SW}}\right)$, magnetic field $(\Delta B)$, and root mean square of irregularities of the magnetic field $\left(\Delta \sigma_{B}\right)$.

\section{Data analysis}

We have investigated the relationships between $\Delta I_{\mu}, \Delta V_{\mathrm{SW}}, \Delta B$ and $\Delta \sigma_{B}$ by the regression analysis. The preparation procedure of the requisite data set is the same as a previous analysis in the paper of Kojima et al. (2015) [2]. In the previous analysis, we only have used the data of $\Delta I_{\mu}$ and $\Delta V_{\mathrm{SW}}$ during 6 years from 2000 to 2005 . In this analysis, we have added the data of $\Delta B$ and $\Delta \sigma_{B}$, and analyzed the all daily means obtained during 17 years from 2000 to 2016 . In order to remove slow variations with periods longer than 27-day, such as a 11-year solar cycle, each data set have been processed by using the 27-day high-pass filter, as before.

\subsection{Simple linear regression analysis}

At first, we have performed simple regression analysis to each dependence of $\Delta I_{\mu}$ on $\Delta V_{\mathrm{SW}}$, $\Delta B$, and $\Delta \sigma_{B}$ respectively in Figure1, 2, and 3.

Figure 1 shows the dependence of $\Delta I_{\mu}$ on $\Delta V_{\mathrm{SW}}$. The regression relation between them is expressed with an equation as follows : $\Delta I_{\mu}=\alpha_{1} \Delta V_{\mathrm{SW}}+\beta_{1}$ with a coefficient $\alpha_{1}=-0.0010 \pm$ 0.00005 , this represents a statistical significance of about $20 \sigma$ (one standard deviation).

Figure 2 shows the dependence of $\Delta I_{\mu}$ on $\Delta B$. The regression relation between them is expressed with an equation as follows : $\Delta I_{\mu}=\alpha_{2} \Delta B+\beta_{2}$ with a coefficient $\alpha_{2}=0.0140 \pm 0.0022$, this represents a statistical significance of about $6.4 \sigma$.

Figure 3 shows the dependence of $\Delta I_{\mu}$ on $\Delta \sigma_{B}$. The regression relation between them is expressed with an equation as follows : $\Delta I_{\mu}=\alpha_{3} \Delta \sigma_{B}+\beta_{3}$ with a coefficient $\alpha_{1}=0.0185 \pm 0.0047$, this represents a statistical significance of about $3.9 \sigma$. 


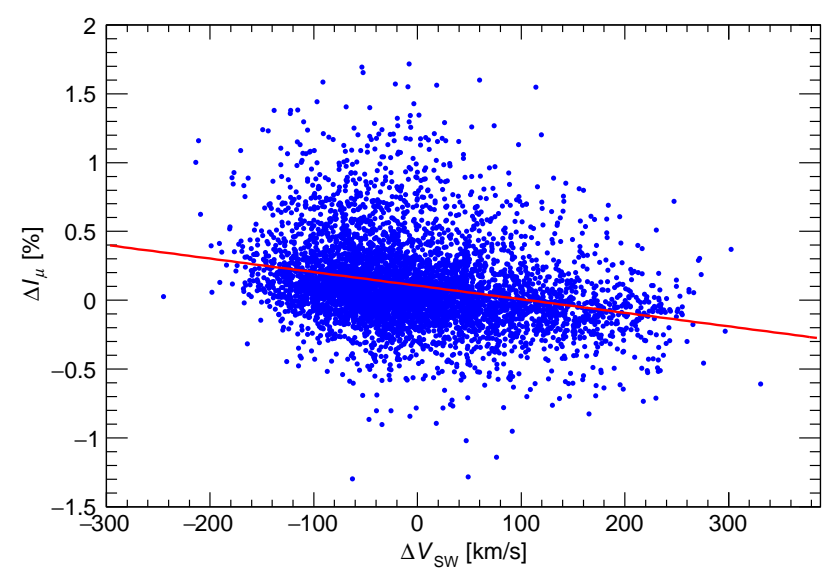

Figure 1: Regression chart of $\Delta I_{\mu}$ vs. $\Delta V_{\mathrm{SW}}$ with a regression line : $\Delta I_{\mu}=\alpha_{1} \Delta V_{\mathrm{SW}}+\beta_{1}$ (red).

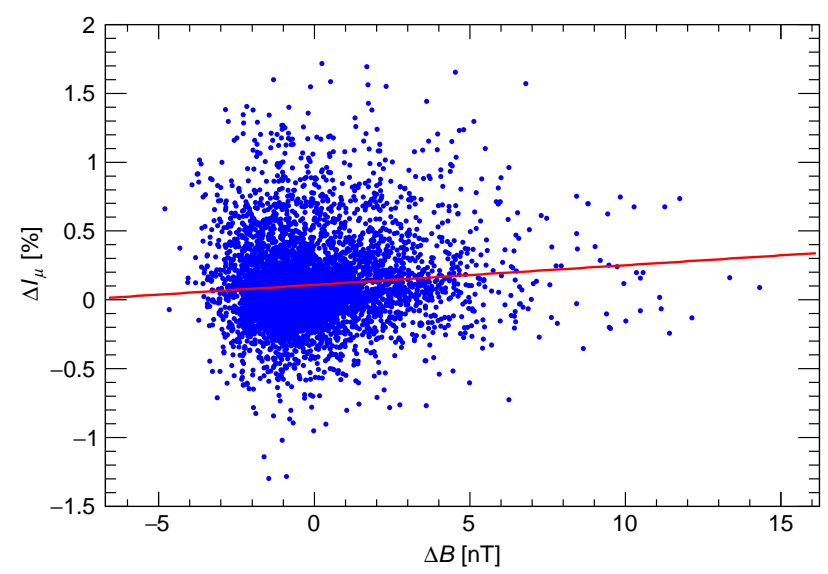

Figure 2: Regression chart of $\Delta I_{\mu}$ vs. $\Delta B$ with a regression line : $\Delta I_{\mu}=\alpha_{2} \Delta B+\beta_{2}$ (red).

From the above results, it is obvious that three quantities we have examined ( $\Delta V_{\mathrm{SW}}, \Delta B$, and $\left.\Delta \sigma_{B}\right)$ are not independent with each other. In order to confirm the dependence between them, the regression relations are shown in Figure4, 5, and 6, below.

Figure 4 shows the relation between $\Delta V_{\mathrm{SW}}$ and $\Delta B$. The regression relation between them is expressed with an equation as follows : $\Delta B=\alpha_{4} \Delta V_{\mathrm{SW}}+\beta_{4}$ with a coefficient $\alpha_{4}=-0.0025 \pm$ 0.0003 , this represents a statistical significance of about $8 \sigma$.

Figure 5 shows the relation between $\Delta V_{\mathrm{SW}}$ and $\Delta \sigma_{B}$. The regression relation between them is expressed with an equation as follows : $\Delta \sigma_{\mathrm{B}}=\alpha_{5} \Delta V_{\mathrm{SW}}+\beta_{5}$ with a coefficient $\alpha_{5}=0.0022 \pm$ 0.0001 , this represents a statistical significance of about $22 \sigma$.

Figure 6 shows the relation between $\Delta B$ and $\Delta \sigma_{B}$. The regression relations between them are expressed with equations as follows : $\Delta \sigma_{B}=\alpha_{6} \Delta B+\beta_{6}$ with a coefficient $\alpha_{6}=0.2836 \pm 0.0050$, this represents a statistical significance of about $57 \sigma$ and $\Delta B=\alpha_{7} \Delta \sigma_{B}+\beta_{7}$ with a coefficient 


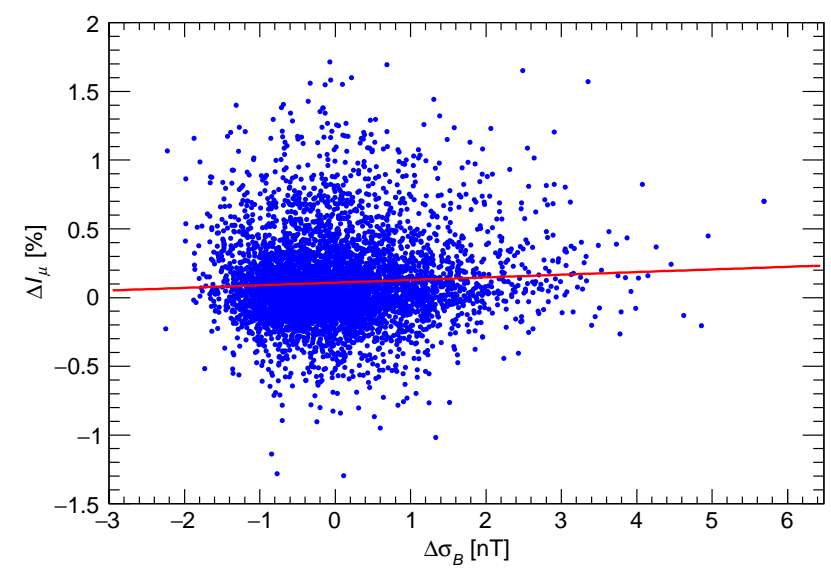

Figure 3: Regression chart of $\Delta I_{\mu}$ vs. $\Delta \sigma_{B}$ with a regression line : $\Delta I_{\mu}=\alpha_{3} \Delta \sigma_{B}+\beta_{3}$ (red).

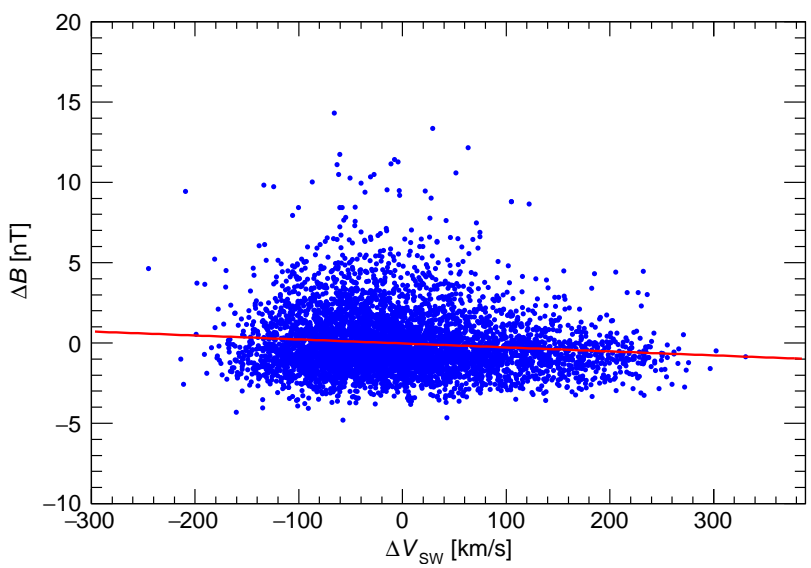

Figure 4: Regression chart of $\Delta B$ vs. $\Delta V_{\mathrm{SW}}$ with a regression line : $\Delta B=\alpha_{4} \Delta V_{\mathrm{SW}}+\beta_{4}$ (red).

$\alpha_{7}=1.3127 \pm 0.0233$, this represents a statistical significance of about $56 \sigma$.

\subsection{Multiple linear regression analysis}

As the results shown in the previous subsection, we have confirmed that three quantities $\left(\Delta V_{\mathrm{SW}}\right.$, $\Delta B$, and $\Delta \sigma_{B}$ ) are not independent with each other. So we have to have examined the dependencies of $\Delta I_{\mu}$ on $\Delta V_{\mathrm{SW}}, \Delta B$, and $\Delta \sigma_{B}$ altogether by a multiple linear regression analysis with an equation of

$$
\Delta I_{\mu}=\alpha_{V} \Delta V_{\mathrm{SW}}+\alpha_{B} \Delta B+\alpha_{\sigma} \Delta \sigma_{B}+\beta_{0} .
$$

and we have obtained three partial regression coefficients as follows :

$$
\begin{aligned}
& \alpha_{V}=-0.0011 \pm 0.00005 \quad \text { with } 22 \sigma \\
& \alpha_{B}=-0.0038 \pm 0.0028 \quad \text { with } 1.4 \sigma
\end{aligned}
$$




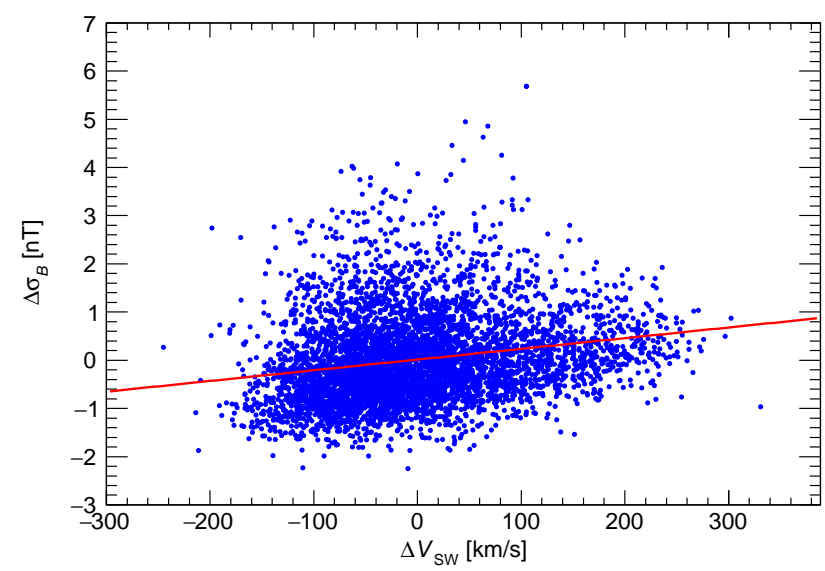

Figure 5: Regression chart of $\Delta \sigma_{B}$ vs. $\Delta V_{\mathrm{SW}}$ with a regression line : $\Delta \sigma_{\mathrm{B}}=\alpha_{5} \Delta V_{\mathrm{SW}}+\beta_{5}$ (red).

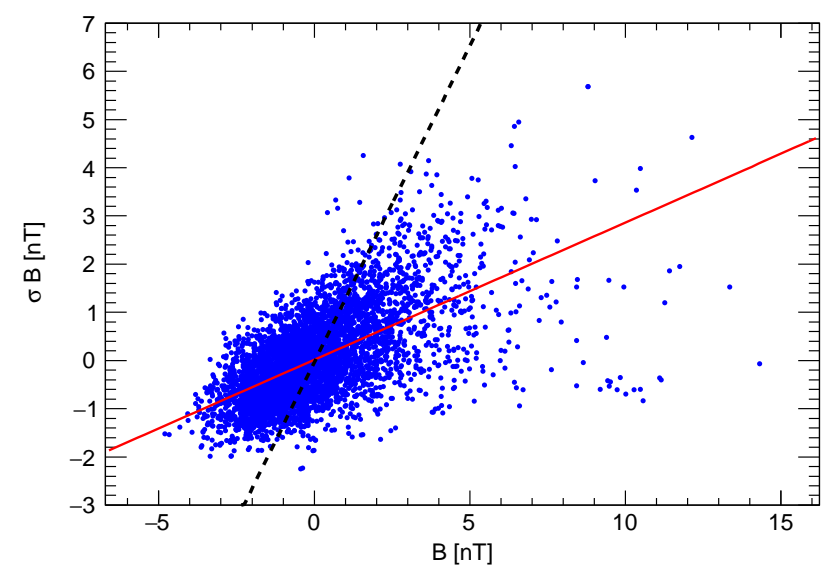

Figure 6: Regression chart of $\Delta \sigma_{B}$ vs. $\Delta B$ with regression lines : $\Delta \sigma_{B}=\alpha_{6} \Delta B+\beta_{6}$ (red) and $\Delta B=$ $\alpha_{7} \Delta \sigma_{B}+\beta_{7}$ (black, dashed).

$$
\text { and } \quad \alpha_{\sigma}=0.0447 \pm 0.0061 \text { with } 7.3 \sigma \text {. }
$$

Standardized partial regression coefficients have also been obtained as $-0.2969,-0.0239$, and 0.1295 respectively.

\section{Results and discussions}

In this paper, we have investigated the relationships between $\Delta I_{\mu}, \Delta V_{\mathrm{SW}}, \Delta B$, and $\Delta \sigma_{B}$ by the regression analysis. The results are summarized in the Table1.

Looking at this table carefully, you can find that the partial regression coefficient of $\Delta I_{\mu}$ on $\Delta V_{\mathrm{SW}}\left(\alpha_{V}\right)$ is almost the same value, with about $20 \sigma$, as the simple regression coefficient between the same pair $\left(\alpha_{1}\right)$. This agreement indicates that the relationship between $\Delta I_{\mu}$ and $\Delta V_{\mathrm{SW}}$ is very 
Table 1: Regression coefficients.

\begin{tabular}{c||c|c|c}
\hline & $\Delta V_{\mathrm{SW}}(\mathrm{km} / \mathrm{s})$ & $\Delta B(\mathrm{nT})$ & $\Delta \sigma_{B}(\mathrm{nT})$ \\
\hline \hline$I_{\mu}(\%)$ & $\alpha_{1}=-0.0010 \pm 0.00005$ & - & - \\
$I_{\mu}(\%)$ & - & $\alpha_{2}=0.0140 \pm 0.0022$ & - \\
$I_{\mu}(\%)$ & - & - & $\alpha_{3}=0.0185 \pm 0.0047$ \\
\hline$I_{\mu}(\%)$ & $\alpha_{V}=-0.0011 \pm 0.00005$ & $\alpha_{B}=-0.0038 \pm 0.0028$ & $\alpha_{\sigma}=0.0447 \pm 0.0062$ \\
\hline \hline$\Delta B(\mathrm{nT})$ & $\alpha_{4}=-0.0025 \pm 0.0003$ & - & - \\
$\Delta \sigma_{B}(\mathrm{nT})$ & $\alpha_{5}=0.0022 \pm 0.0001$ & - & - \\
\hline \hline$\Delta \sigma_{B}(\mathrm{nT})$ & - & $\alpha_{6}=0.2836 \pm 0.0050$ & - \\
$\Delta B(\mathrm{nT})$ & - & - & $\alpha_{7}=1.3127 \pm 0.0233$ \\
\hline
\end{tabular}

robust and almost independent of $\Delta B$ or $\Delta \sigma_{B}$. This fact provides a justification for our previous analysis examined the $\Delta I_{\mu}$ dependence on $\Delta V_{\mathrm{SW}}$ [2].

Meanwhile, the other two partial regression coefficients $\left(\alpha_{B}\right.$ and $\left.\alpha_{\sigma}\right)$, with $1.4 \sigma$ and $7.3 \sigma$ respectively, are somewhat different from the simple regression coefficients $\left(\alpha_{2}\right.$ and $\left.\alpha_{3}\right)$, with $6.4 \sigma$ and $3.9 \sigma$ respectively. In addition the sign of $\alpha_{B}$ (negative) is different from the sign of $\alpha_{2}$ (positive), even they represent the same depdndence of $\Delta I_{\mu}$ on $\Delta B$. From these fact and from the rather strong correlation between $\Delta B$ and $\Delta \sigma_{B}$ suggested by $\alpha_{6}$ and $\alpha_{7}$, we cannot deduce any definitive result about the dependence of $\Delta I_{\mu}$ on $\Delta B$ and $\Delta \sigma_{B}$ at this stage of investigation.

In this paper, we conclude that the observed variations of cosmic ray intensity would mainly be caused by the variation of solar wind speed. The dependence of the observed variations of cosmic ray intensity on the variation of IMF strength or irregularity is a remaining issue.

\section{Acknowledgments}

Use of the OMNI data at the GSFC/SPDF OMNIWeb [8] was important for our analyses for which we are grateful to the omni group.

\section{References}

[1] Y. Hayashi et al., A large area muon tracking detector for ultra-high energy cosmic ray astrophysics: The GRAPES-3 experiment, Nucl. Instrum. Methods Phys. Res. A 545 (2005) 643.

[2] H. Kojima et al., Dependence of cosmic ray intensity on variation of solar wind velocity measured by the GRAPES-3 experiment for space weather studies, Phys. Rev. D 91 (2015) 121303(R).

[3] D. B. Swinson, 'Sidereal' Cosmic-Ray Diurnal Variations, J. Geophys. Res. 74 (1969) 5591. D. B. Swinson, Field Dependent Cosmic Ray Streaming at High Rigidities, J. Geophys. Res. 81 (1976) 2075.

[4] S. Yasue, North-South Anisotropy and Radial Density Gradient of Galactic Cosmic Rays, J. Geomag. Geoelectr. 32 (1980) 617.

[5] H. Kojima et al., Measurement of the radial density gradient of cosmic ray in the heliosphere by the GRAPES-3 experiment, Astropart. Phys. 62 (2015) 21. 
[6] E. N. Parker, Cosmic-Ray Modulation by Solar Wind, Phys. Rev. 110 (1958) 1445.

[7] H. Kojima et al., Measurement of the radial diffusion coefficient of galactic cosmic rays near the Earth by the GRAPES-3 experiment, Phys. Rev. D 98 (2018) 022004.

[8] http://omniweb.gsfc.nasa.gov 